\title{
Generation of laser radiation by nanostructured solid active elements with selective optical nanoresonators formed in nanoporous aluminum oxide films
}

\author{
G. A. Lyubas \\ Vorozhtsov Institute of Organic Chemistry, Siberian Branch, Russian Academy of Sciences, \\ Novosibirsk, 630090 Russia \\ sciencenano@yandex.ru
}

\author{
PACS 82.45.Cc; 82.45.Aa; 82.45.Yz; 81.15.Pq; 81.07.-b; 33.50.-j; 78.45.+h; 42.55.Mv; 77.55.+f \\ DOI 10.17586/2220-8054-2017-8-6-793-797
}

\begin{abstract}
The generation of laser radiation by a nanostructured solid active element with natural selective optical nanoresonator formed in a nanoporous aluminum oxide film activated with rhodamine $6 \mathrm{G}$ has been obtained for the first time. The lasing is characterized by high-quality radiation with the absence of a spontaneous component. Chemical deposition of noble metals leads to the formation of internal nanoresonator into the porous structure. This reduces generation threshold more than two fold.
\end{abstract}

Keywords: nanoporous aluminum oxide films, electrochemical and chemical metallization, nanoparticles, nanostructured solid active elements, generation of laser radiation.

Received: 30 November 2017

Revised: 8 December 2017

\section{Introduction}

The development of solid lasers based on organic compounds and, in particular, the use of nanoporous aluminum oxide (NAO) as the active medium activated with rhodamine $6 \mathrm{G}$ is a topical problem of modern material engineering [1]. There is the possibility of creating compact microlasers based on them, which will find wide application in various spheres of science and engineering [2,3]. At present, research concerning the creation of laser generation using nanostructured materials activated with organic laser dies is being actively carried out [4-6], in particular, with the use of aluminum oxide porous ceramics [7]. The studies of fluorescent properties of rhodamine dies incorporated into aluminum oxide pores were carried out in [8-10], where it was shown that the fluorescence intensity of rhodamine $6 \mathrm{G}$ dye in aluminum oxide matrix increased compared to that in porous glass. The induced emission in the geometry to transmission in a porous matrix of anodic aluminum oxide doped with rhodamine 6G was obtained in [11]. The induced emission was observed against the background of a dye spontaneous radiation. The possibilities of generation in other modes and the ways to improve the quality were not considered. The generation of laser radiation by nanostructured solid active elements based on NAO films activated with rhodamine $6 \mathrm{G}$ in the geometry to reflection was obtained in [12].

This work was aimed at obtaining the generation of laser radiation by solid active elements with selective nanoresonators formed in the nanoporous structures.

\section{Experimental}

Aluminum plates with a thickness of $0.1 \mathrm{~mm}$, highpurity grade of $99.95 \% \mathrm{AL}$, and size of $50 \times 50 \mathrm{~mm}^{2}$ were taken for anodization. Before anodizing, the plates were purified by de-oiling in $10 \%$ solution of $\mathrm{NaOH}$ and blooming in $10 \%$ solution of nitric acid. Then the material was electrochemically polished in a solution of the following composition: orthophosphoric acid (relative density of 1.7) $34 \mathrm{~g}$, sulfuric acid (relative density of 1.84) $34 \mathrm{~g}$, chromic anhydride $4 \mathrm{~g}$, and water to $100 \mathrm{~g}$. The electropolishing mode was $U_{\text {post }}=12 \mathrm{~V}$, current density was $2.5 \mathrm{~A} / \mathrm{dm}^{2}$, temperature was $80-90{ }^{\circ} \mathrm{C}$, and duration was $2-3 \mathrm{~min}$. The anodization of the samples was carried out in 2 and $20 \%$ solutions of sulfuric acid at $20{ }^{\circ} \mathrm{C}, U_{\text {post }}=12 \mathrm{~V}$, and current density of $2 \mathrm{~A} / \mathrm{dm}^{2}$ in potentiostatic mode. The anodization parameters are described more detail in [13-21]. The anodizing duration amounted from 10 to $300 \mathrm{~min}$. For the chosen mode and duration of aluminum anodization, layers from $\approx 200 \mathrm{~nm}$ to $\approx 85 \mu \mathrm{m}$, respectively, were formed on its surface.

Deposition of copper particles was performed electrochemically by the current of alternating polarity $U_{\text {alter }}=12-14 \mathrm{~V}$ and a current density of $0.2-0.5 \mathrm{~A} / \mathrm{dm}^{2}$ (potentiostatic regime) from an electrolyte with the following composition: copper sulfate $50 \mathrm{~g} / \mathrm{L}$, magnesium sulfate $20 \mathrm{~g} / \mathrm{L}$, and sulfuric acid (up to $\mathrm{pH}=1$ ). Treatment time was 3-4 min. Further treatment of layers containing copper nanoparticles in solutions of silver nitrate 
and gold tetrachloride to form silver and gold nanoparticles by exchange reactions was done from solutions of the following composition: for $\mathrm{Ag}$, silver nitrate $1 \mathrm{~g} / \mathrm{L}$, sulfuric acid (to $\mathrm{pH}=2$ ); for $\mathrm{Au}$, chloroauric acid $\left(\mathrm{HAuCl}_{4}\right)$ $1 \mathrm{~g} / \mathrm{L}$, sulfuric acid (up to $\mathrm{pH}=1-2$ ). Treatment time was 3-4 min.

The adsorption of rhodamine $6 \mathrm{G}$ molecules on NAO films in most cases was carried out from a solution with a concentration $C=10^{-3} \mathrm{~mol} / \mathrm{L}$, which is the optimal value.

In order to obtain the images of pores with a superhigh resolution, we used a Zeiss MERLIN VP Compact scanning electron microscope with a resolution of $5 \mathrm{~nm}$, which works at $20 \mathrm{kV}$.

In order to measure the laser-generation spectra, we used an Avantas (AvaSpec-1024) spectrometer with a resolution of $0.4 \mathrm{~nm}$ in a wavelength range of $235-735 \mathrm{~nm}$. In the pulsed mode, we used an Nd:YAG laser LOTIS TII (model LS2147) as a source of the laser generation excitation in the samples. The measurements were carried out by single pulses on the second harmonic (wavelength $\lambda_{e x}=532 \mathrm{~nm}$ ), pulse energy $E$ from 0 to $90 \mathrm{~mJ}$, and pulse duration $t=20 \mathrm{~ns}$.

\section{Results and discussion}

\subsection{Formation of selective optical nanoresonators in the NAO films}

Formation of nanoresonators in the NAO films is explained by the specificity of chemical deposition; as a result, an additional effective layer of noble metal is formed on the surface of NAO, which leads to the formation of the film nanostructure of NAO, like the structure of a Fabry-Perot nanointerferometer (Fig. 1).

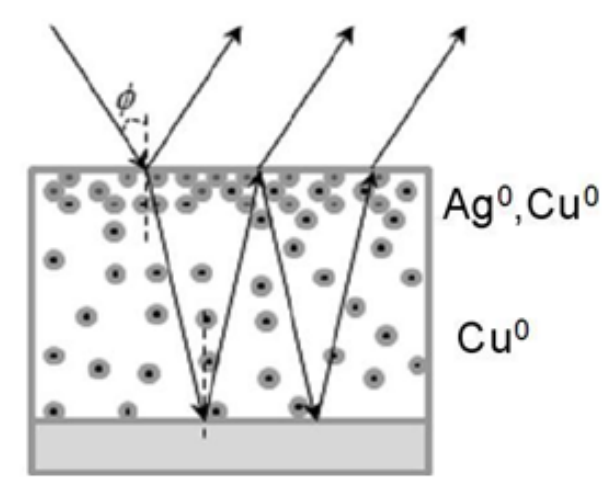

FIG. 1. Scheme of selective nanoresonator in the NAO film, similar to the structure of FabryPerot nanointerferometer

According to electron microscopy studies (see Fig. 2), alumina films have a high pore density. The pores are close to each other, have a wall thickness from 7 to $10 \mathrm{~nm}$ and a diameter of about 9-14 $\mathrm{nm}$. As can be seen from the Fig. 2, the NAO films have the hexagonal (honeycomb) structure of arrangement of pores. The formed silver nanoparticles have a diameter from $\approx 5$ to $\approx 85 \mathrm{~nm}$ and are located predominantly on the surface or near the upper boundary of the porous layer (see Fig. 2). Silver nanoparticles being reduced on copper inside the pores are released from the pores and localized on the surface. According to the general opinion, metal electrodeposition in NAO occurs within the alumina pores and starts at the bottom part of pores [22]. However, according to the electron-microscopic image (Fig. 2), during the chemical deposition, silver nanoparticles cover open ends of pores on the surface of the film. The process of directed diffusion migration of silver nanoparticles during chemical deposition of the latter can explain this fact. Silver cations are reduced within the pores on copper particles, copper being oxidized to the monovalent state. At the same state, intermediate solvated contact pairs of cations $\mathrm{Cu}^{+}$ bonded to an $\mathrm{Ag}^{0}$ atom are formed in a NAO narrow pore with a diameter of about 9-14 nm. Since these pairs have the same positive charge, the Coulomb repulsion facilitates their directional diffusion from the bottom of the pore to its surface. This leads to the formation of nanoparticles of noble metals predominantly on the surface of the NAO film or near it. Possible chemical reactions are shown on the scheme below:

$$
\begin{gathered}
\mathrm{Cu}^{0}+\mathrm{Ag}^{+} \longrightarrow\left(\mathrm{Cu}^{+}, \mathrm{Ag}^{0}\right)_{\text {solv }} \\
\left(\mathrm{Cu}^{+}, \mathrm{Ag}^{0}\right) \stackrel{\text { diffusion }}{ }\left(\mathrm{Cu}^{+}, \mathrm{Ag}^{0}\right) \mathrm{n}_{\text {solv }} \\
\left(\mathrm{Cu}^{+}, \mathrm{Ag}^{0}\right) \mathrm{n}_{\text {solv }} \longrightarrow \mathrm{Ag}_{n}^{0}+\mathrm{Cu}_{n}^{+} \\
\mathrm{Cu}_{n}^{+}+\mathrm{Ag}_{n}^{+} \longrightarrow \mathrm{Cu}_{n}^{+2}+\mathrm{Ag}_{n}^{0}
\end{gathered}
$$




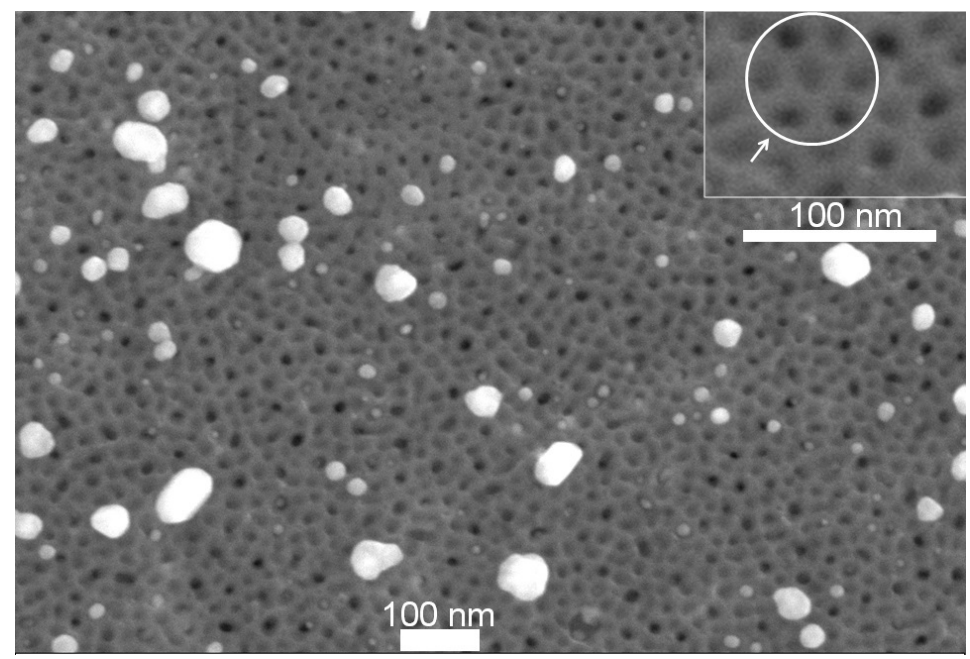

FIG. 2. Electron microscopic photograph of superhigh resolution of the film of NAO (top view; hexagonal structure of arrangement of pores)

$$
\mathrm{Cu}_{n}^{+} \longrightarrow \mathrm{Cu}_{n}^{0}+\mathrm{Cu}_{n}^{+2} \text {. }
$$

Reactions (3)-(5) occur on the film surface. Disproportionation of intermediate $\mathrm{Cu}^{+}$cations again yields $\mathrm{Cu}^{0}$ atoms (step 5), which actively reduce silver cations not inside but on the surface of the pores and reactions (1)-(5) repeated with subsequent aggregation of silver nanoparticles on the surface of the NAO film. Similar reactions occur in the case of chemical deposition of gold. Thus, the process of chemical deposition of noble metals on NAO with electrochemically predeposited copper nanoparticles leads to a redistribution of nanoparticles of noble metal from the bottom and walls to the top part of pores. A thin surface layer enriched with Ag or Au nanoparticles makes a high contribution to the imaginary part of the complex refractive index due to the nature of the Plasmon absorption of light by nanoparticles; therefore, the reflection coefficient of the surface layer increases as compared with a pure NAO film. A nanoresonator in which practically ideal conditions are created for multiple beam passing is formed automatically. Formation of the nanostructure of such a Fabry-Perot nanointerferometer increases the number of rereflections within the layer of NAO film, which increases the gain of active medium (active medium is NAO film activated with rhodamine 6G). As can be seen from the Fig. 2, large silver nanoparticles are distributed on the surface of NAO at the distance of about 30-250 nm; i.e., the film is not continuous and pores are available for penetration of dye (rhodamine $6 \mathrm{G}$ ) molecules.

In our case, the phenomenon of the formation of the surface film of noble metal is unusual because it occurs spontaneously by redistributing the metal nanoparticles from the bottom of pore to its surface during the chemical reduction of a salt of noble metal on the electrochemically metallized NAO. Technically, the process of applying of the surface metal film by immersing of the metallized NAO film in a solution of noble metal salt is considerably simpler to perform than to carry out the vacuum deposition of a metal film.

The reflection spectra of the alumina films with deposited silver (solid line) and gold (dotted line) nanoparticles in reference to aluminum mirror are shown in Fig. 3. It can be seen the effective reflection region for silver and gold is observed at $\lambda_{e m}=572 \mathrm{~nm}$ (a wavelength of the generated laser radiation). From Fig. 3 it is evident that gold and silver have a relatively effective selectivity. In the case of gold the wavelength of $572 \mathrm{~nm}$ is reflected in $\sim 2.4$ times better than a wavelength of $532 \mathrm{~nm}$; i.e., $\mathrm{R}(\mathrm{Au})_{572 \mathrm{~nm}} / \mathrm{R}(\mathrm{Au})_{532 \mathrm{~nm}} \approx 2.4$, where $\mathrm{R}$ is the reflection intensity. With silver the situation is similar; $\mathrm{R}(\mathrm{Ag})_{572 \mathrm{~nm}} / \mathrm{R}(\mathrm{Ag})_{532 \mathrm{~nm}} \approx 2.5$. This allows the use of nanoparticles of gold and silver as the mirrors of the nanoresonator for amplifying of the generated laser radiation $\left(\lambda_{e m}=572 \mathrm{~nm}\right)$.

\subsection{Generation of laser radiation by NAO film with selective optical nanoresonator formed in the porous structure}

In the absence of an external resonator, the generation of laser radiation by NAO film with natural selective nanoresonator formed into the porous structure was obtained. Fig. 4 shows the spectrum of laser radiation generation by the film of NAO activated with rhodamine $6 \mathrm{G}$ with internal nanoresonator. The generation occurs at a wavelength of $572 \mathrm{~nm}$ The line half-width is narrow and amounts to $\Delta \lambda_{F W H M}=9 \mathrm{~nm}$. It is important to note that a component of spontaneous radiation is completely absent in the induced radiation spectrum. The 


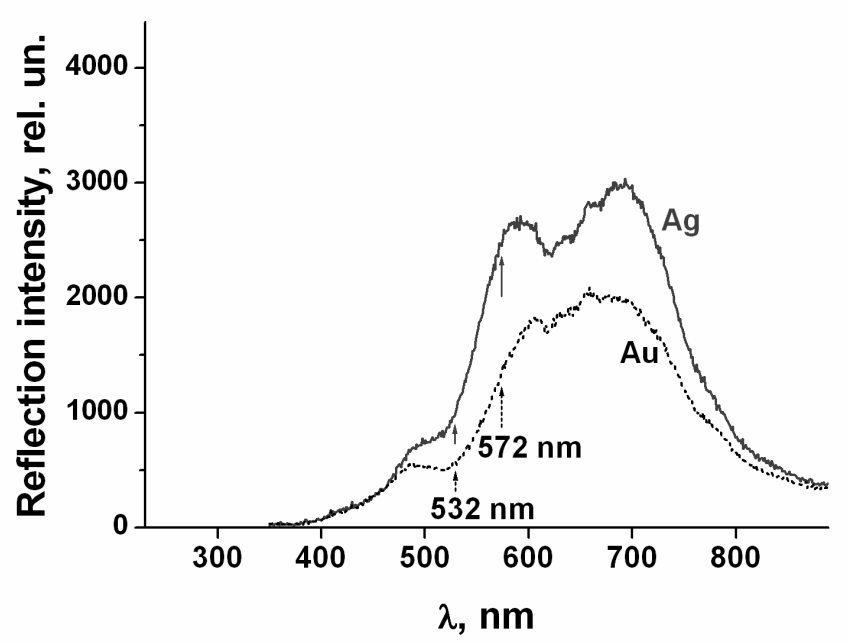

FIG. 3. Reflection spectra of the alumina films with deposited silver (solid curve) and gold (dotted curve) nanoparticles

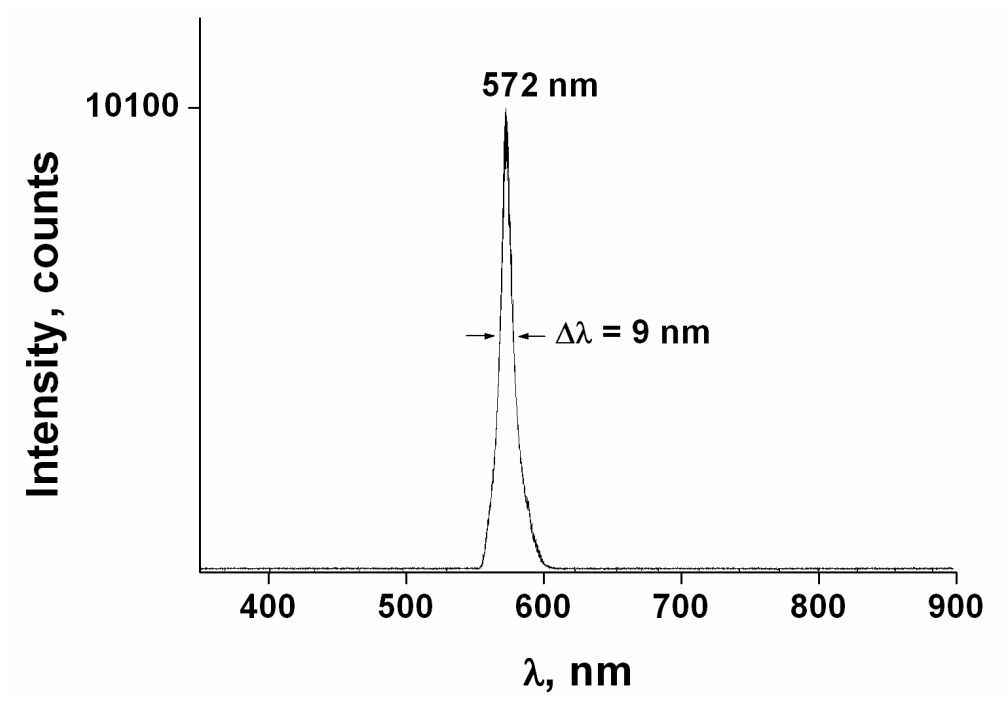

FIG. 4. Spectrum of laser radiation generation by the film of NAO activated with rhodamine $6 \mathrm{G}$ with internal natural nanoresonator

absence of a spontaneous radiation component indicates the efficient pumping of the energy from the contour of the luminescence spectrum into a line of generation, which, for example, was not achieved in [11]. The transition from the photoluminescence to laser generation by the full absence of a spontaneous component occurs at a value of impinging radiation power density of about $P w \sim 1 \mathrm{MW} / \mathrm{cm}^{2}$ By taking into account small sample thickness and low quality of the internal nanoresonator, this value can be considered small.

\section{Conclusion}

Thus, in this work we have obtained and studied the generation of laser radiation by nanostructured solid active elements based on the films of nanoporous aluminum oxide with natural selective optical nanoresonators. Chemical deposition of noble metals leads to a redistribution of nanoparticles from the bottom to the top part of the nanoporous layer. This method achieves a sharper Fabry-Perot interference in a layered structure and reduces generation threshold more than two fold. The experiments have shown that the value of the generation threshold decreases to $\sim 1 \mathrm{MW} / \mathrm{cm}^{2}$, when nanoresonator is formed into the porous structure. In the absence of an external resonator, the generation of laser radiation with natural selective optical internal nanoresonator is obtained for the 
first time. In this case, the background of spontaneous luminescence is completely absent in the laser generation. The absence of a spontaneous radiation component indicates the efficient pumping of the energy from the contour of the luminescence spectrum into a line of generation. As a rule, this is inherent in solid lasers, like Nd:YAG, but in our case this phenomenon is observed in the solid element activated with an organic dye, which indicates the high quality of the obtained nanostructured solid elements approaching solid lasers in their properties.

Possible applications include micro/nanoscale lasers with indirect electrical pumping by laser diodes.

\section{References}

[1] Zemskii V.I., Kolesnikov Yu.L., and Meshkovskii I.K. Physics and Technology of Pulsed Dye Lasers. SPbGU ITMO, St. Petersburg, 2005, 176 p. [in Russian].

[2] Zhang W., Yao J., Zhao Y.S. Organic Micro/Nanoscale Lasers. Acc. Chem. Res., 2016, 49(9), P. 1691-1700.

[3] Li Y.J., Yan Y., Zhao Y.S., Yao J. Construction of Nanowire Heterojunctions: Photonic Function-Oriented Nanoarchitectonics. Adv. Mater., 2016, 28, P. 1319-1326.

[4] Bol'shchikov F.A., Garibin E.A., Gusev P.E., Demidenko A.A., Kruglova M.V., Krutov M.A., Lyapin A.A., Mironov I.A., Osiko V.V., Reitirov V.M. Ryabochkina P.A., Sakharov N.V., Smirnov A.N., Ushakov S.N., and Fedorov P.P. Nanostructured Tm:CaF 2 ceramics: potential gain media for two micron lasers. Quantum Electron., 2011, 41, P. 193.

[5] Shin H.W., Cho S.Y., Choi K.H., and Kim Y.R. Directional random lasing in dye- $\mathrm{TiO}_{2}$ doped polymer nanowire array embedded in porous alumina membrane. Appl. Phys. Lett., 2006, 88, P. 263112.

[6] Costel A., Garcia-Moreno I., del Agua D., García O., and Sastre R. Highlyphotostable solid-state dye lasers based on silicon-modified organic matrices. J. Appl. Phys., 2007, 101(7), P. 731-742.

[7] Marinho S.J., Jesus L.M., Barbosa L.B., Ardila D.R., Alencar M., Rodrigues J.J. Bi-chromatic random laser from alumina porous ceramic infiltrated with rhodamine B. Jr. Laser Phys. Lett., 2015, 12, P. 055801-055805.

[8] Moadhen A., Elhouichet H., Nosova L., Oueslati M. Rhodamine B absorbed by anodic porous alumina: Stokes and anti-Stokes luminescence study. J. Lumin., 2007, 126(2), P. 789-794.

[9] Li Z., Huang K. Optical properties of alumina membranes prepared by anodic oxidation process. J. Lumin., 2007, 127(2), P. 435-440.

[10] Zhang Z.L., Zheng H.R., Dong J., Yan X.Q., Sun Y., Xu H.X. Surface enhanced fluorescence by porous alumina with nanohole arrays. Sci.China, Ser. G., 2012, 55(5), P. 767-771.

[11] Ibrayev N.Kh., Zeinidenov A.K., Aimukhanov A.K. and Napolskii K.S. Stimulated emission from aluminium anode oxide films doped with rhodamine 6G. Quantum Electron., 2015, 45, P. 663

[12] Lyubas G.A. Generation of laser radiation by nanostructured solid active elements based on nanoporous aluminum oxide films activated with rhodamine 6G. Nanotechnologies in Russia, 2017, 12(5-6), P. 276-284.

[13] Lyubas G.A., Shelkovnikov V.V., and Korotaev S.V. Optical interferometric sensor based on thin layers of nanoporous anodized aluminum containing nanoparticles of noble metals. Nanotechnologies in Russia, 2016, 11(1-2), P. 29-40.

[14] Shelkovnikov V.V., Lyubas G.A., and Korotaev S.V. Enhanced reflective interference spectra of nanoporous anodic alumina films by double electrochemical deposition of chemical metal nanoparticles. Prot. Met. Phys. Chem. Surf., 2016, 52(2), P. 227-231.

[15] Shelkovnikov V.V., Lyubas G.A., Korotaev S.V. Controlled interference color of the metal surface by combination of the chemical and electrochemical aluminum surface treatment. Nanosystems: Physics, Chemistry, Mathematics, 2014, 5(5), P. 718-727.

[16] Kumeria T., Rahman M.M., Santos A., Ferré-Borrull J., Marsal L.F., Losic D. Structural and optical nanoengineering of nanoporous anodic alumina rugate filters for real-time and label-free biosensing applications. Anal. Chem., 2014, 86, P. 1837-1844.

[17] Santos A., Kumeria T., Losic D. Nanoporous Anodic Alumina: A Versatile Platform for Optical Biosensors. Materials, 2014, 7, P. 42974320.

[18] Santos A., Kumeria T., Losic D. Optically optimized photoluminescent and interferometric biosensors base on nanoporous anodic alumina: A comparison. Anal. Chem., 2013, 85, P. 7904-7911.

[19] Ferré-Borrull J., Rahman M.M., Pallares J., Marsal L.F. Tuning nanoporous anodic alumina distributed-Bragg reflectors with the number of anodization cycles and the anodization temperature. Nanoscale Research Letters, 2014, 9, P. 416-422.

[20] Ferré-Borrull J., Pallares J., Macias G., Marsal L.F. Nanostructural Engineering of Nanoporous Anodic Alumina for Biosensing Applications. Materials, 2014, 7, P. 5225-5253.

[21] Macias G., Hernández-Eguía L.P., Ferré-Borrull J., Pallares J., Marsal L.F. Gold-coated ordered nanoporous anodic alumina bilayers for future label-free interferometric biosensors. ACS Appl. Mater. Interfaces, 2013, 5, P. 8093-8098.

[22] Hwang S.K., Jeong S.H., Lee O.J., Lee K.H. Fabrication of vacuum tube arrays with a submicron dimension using anodic aluminum oxide nanotemplates. Microelectronic Engineering, 2005, 77, P. 2-7. 